\title{
The safe use of the transvaginal ultrasound probe for transabdominal oocyte retrieval in patients with vaginally inaccessible ovaries
}

\author{
Baldini $\mathrm{D}^{1 *}$, Lavopa $\mathrm{C}^{1}$, Vizziello $\mathrm{G}^{1}$, Sciancalepore $\mathrm{AG}^{1}$ and Malvasi $\mathrm{A}^{2}$ \\ ${ }^{1}$ Momò Fertilife Clinic, Bisceglie, Italy \\ ${ }^{2}$ Santa Maria Hospital, Bari, Italy
}

\begin{abstract}
Objective: The aim of this study was to compare the complications of the oocyte retrieval, the number of oocytes obtained and the intra-cytoplasmic semen injection (ICSI) outcomes in the same patients undergoing both transvaginal and transabdominal ultrasound-guided approaches, by using the same vaginal ultrasound probe.

Methods: 1972 oocyte pick up were reviewed in the period between January 2012 and December 2017, among which were identified 21 women, in which both transabdominal and transvaginal approaches were performed by using the same ultrasound vaginal probe. For each patient, the number of retrieved, mature and degenerated oocytes were compared between the transvaginal and transabdominal approach. Intra e post-operative procedure complications were also evaluated.

Results: The number of retrieved, mature and degenerated oocytes were very similar, independently by the retrieval method used. Furthermore, for all patients and independently by the retrieval method used, neither relevant intra-operative complications nor postoperative infections were noticed.

Conclusions: This study points out that the oocyte retrieval procedure did not influence the outcome of pick up and evidenced the absence of significant complications for the transabdominal ultrasound-guided procedure. The use of the transvaginal ultrasound probe provides a controlled framework of examination and highresolution images. Overall, even the transvaginal approach remains the favored choice for oocyte retrieval, the results of this study strengthen the adoption of the transabdominal aspiration in situations where one of both ovaries is not accessible.
\end{abstract}

\section{Introduction}

The oocyte retrieval, also called oocyte pick up, is a crucial surgical route in the IVF cycle which affects the outcome of Assisted Reproductive Technology (ART). Historically, the oocyte pick up was carried out by laparoscopy $[1,2]$. It usually takes the insertion of three instruments into the woman's abdomen by three small incisions. The first incision permits to insert the laparoscope at the lower edge of the navel, thus enabling the operator to see the ovaries and the follicles containing the oocytes. The second small incision is usually made on one side of the abdomen to insert an instrument to hold the ovaries in place. The needle for removing the eggs is inserted through a third small incision, usually at one side of the abdomen. Laparoscopic oocyte retrieval has been progressively abandoned as it is a long and quite complex procedure, which may cause pain, bruising and itching also after several days $[3,4]$. Moreover, it exposes patients to severe risks for example infections, internal organ damages or bleeding and finally results in low rates of mature oocyte recovery and fertilization [5].

To overcome such shortcomings, transvaginal ultrasound-guided follicle aspiration has been progressively adopted as the standard procedure of oocyte harvesting $[1,6]$. It consists in the insertion of a small needle with an attached ultrasound probe into the vagina which is slightly moved by means of the top wall of the vagina. The ultrasound probe allows to visualize on the ultrasound screen the follicles, then the needle is inserted into every follicle to extract the follicular fluid and collect it into a tube $[7,8]$. The transvaginal ultrasound-guided follicle aspiration is usually the preferable process for oocyte pick up since it is fast (it takes about $20 \mathrm{~min}$ ), not invasive and requires minimal anesthesia and recovery time. Furthermore, it is a highly reproducible tool for oocyte retrieval.

Nevertheless, the transvaginal approach becomes unfeasible if the ovaries of infertile patients are not easily accessible by transvaginal ultrasonography, due to changes in the anatomy of pelvic organs which may be caused, for example, by biological variations or pelvic disease [9]. In these cases, the oocyte retrieval should be performed by an alternative procedure, such as the transabdominal ultrasound-guided follicular aspiration [10-13]. This approach is particularly useful in the case of patients with ovarian malposition [14,15]. Previous reports have been published using the transabdominal retrieval method [16,17] but the analysis of fertilization results has been performed on two distinct groups of patients. On the contrary, the aim of this study was to compare the complications of the oocyte retrieval, the number of oocytes obtained and ICSI outcomes in the same group of patients undergoing both transvaginal and transabdominal ultrasound-guided approaches, by using the same vaginal ultrasound probe also for the transabdominal oocyte retrieval.

${ }^{\star}$ Correspondence to: Domenico Baldini, Momò Fertilife Clinic, Bisceglie, Italy E-mail: dbaldini@libero.it

Key words: intra-cytoplasmic semen injection (ICSI), oocyte retrieval, transabdominal follicular aspiration, transvaginal follicular aspiration, transvaginal probe

Received: April 30, 2018; Accepted: May 17, 2018; Published: May 21, 2018 


\section{Materials and Methods}

1972 ICSI cycles were reviewed in the period between January 2012 and December 2017, among which were identified 21 women who had underwent contemporarily both transvaginal and transabdominal ultrasound-guided follicle aspiration. Namely, oocytes retrieval was performed by transvaginal aspiration in one ovary and via transabdominal aspiration in the contralateral ovary.

Couples were treated for infertility at the Momò Fertilife Private Center for Reproductive Medicine, in Bisceglie (Italy) and gave written inform consent to participate. During follicular monitoring of those patients, only one ovary was accessible vaginally while the contralateral ovary was not visualized vaginally or was seen more easily abdominally. The semen quality of the study patients' partners had normal parameters according to the World Health Organization.

Women were treated with a conventional gonadotropin-releasing hormone (GnRH) antagonist (Cetrotide, Merck Serono) and stimulated with a recombinant Follicle Stimulating Hormone (FSH) preparation (GONAL-f, Merck Serono). Semen was collected in sterile containers by masturbation after $3 / 4$ days of sexual abstinence and then maintained at $37^{\circ} \mathrm{C}$ for $30 \mathrm{~min}$. After liquefaction, samples were analyzed for sperm concentration, motility and morphology according to the World Health Organization criteria.

To carry out the oocyte retrieval, patients were placed in the dorsal supine position and underwent procedural sedation with intravenous Propofol. Transvaginal oocyte retrieval was performed through follicular puncture by a 17-gauge retrieval needle (SOMATEX Medical Technologies) under the guidance of an ultrasound probe (VOLUSON S8, GE Healthcare) attached to a sterile needle guide. The probe equipped with a disposable cover and placed into the vagina. When transvaginal aspiration was unfeasible for one ovary, it was attempted to reach the ovary transvaginally by standard procedures for example by applying an abdominal pressure to push ovaries into the pelvis. If such procedure failed, the same operator carried out the transabdominal aspiration to the patient subjected to the same conscious sedation used for transvaginal aspiration. To this aim, patient abdomen was treated with a betadine solution, which was washed off with physiological solution and dried with sterile dressing. The operator moved the same transvaginal ultrasound probe over the abdomen in correspondence to the ovary and the retrieval needle coupled with a needle guide was inserted through the abdomen skin (Figure 1). In all patients, the retrieval of oocyte was obtained by a single ovarian puncture. There were no failed attempts.

Oocytes were collected at $36 \mathrm{~h}$ post-human Chorionic Gonadotropin (hCG) administration and divided into two equal groups based on the retrieval method used. Cumulus-oocyte complexes were exposed to Hyaluronidase solution $(25 \mathrm{IU} / \mathrm{ml})$ to remove by pipetting the corona radiata. Metaphase II oocytes were evaluated and select under a stereomicroscope (Nikon SMZ 1500). Only oocytes in metaphase II were injected. The oocytes were incubated in LGGF medium (Fertilization Global) and injected 38-40 hours after hCG administration. In both groups, the Intra-Cytoplasmic Semen Injection (ICSI) procedure was performed on heated stage at $37^{\circ} \mathrm{C}$ under an inverted microscope (Nikon eclipse TE 200) at 400X magnification. ICSI procedure was performed by oil-hydraulic microinjection system (Nikon eclipse TE 200). Normal fertilization was defined as zygotes with 2 pronuclei, then fertilized oocytes were continuously cultured in LGGG medium (Global) for 3 days.

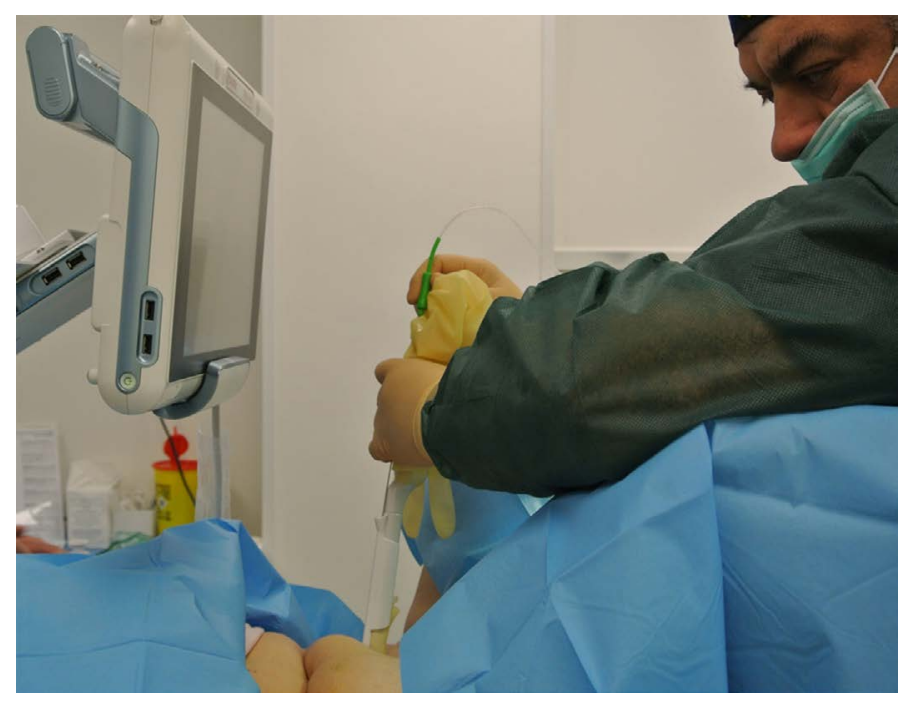

Figure 1: Transabdominal ultrasound-guided follicular aspiration in progress

For each patient, the number of oocytes retrieved, the oocyte quality, the fertilization rate were compared between the transvaginal and transabdominal approach used for oocyte retrieval. Procedure complications were evaluated.

Data are expressed as mean \pm standard deviation for continuous variables, while percentages were used for categorical variables. Data analysis was performed by using Statistica version 8.0 (StatSoft Italia Srl, Padova). Mean values were compared by Student's $t$ test. ( $p$ value $<0.05$ ) was considered significant. All patients were included once for the analysis.

\section{Results and Discussion}

The transvaginal ultrasound-guided follicular aspiration has been worldwide adopted as the standard approach for oocyte retrieval, due to its safety, reproducibility and efficiency [18]. Nevertheless, in patients with ovaries not accessible transvaginally, clinicians should select another method, mostly consisting in the transabdominal ultrasound guided aspiration.

Previous reports have compared these approaches and the results of IVF outcomes in two distinct groups of patients [19] based on similar, but not identical parameters. On the contrary, this study was focused on the comparison between the transvaginal and transabdominal oocyte retrieval performed in the same patients. The criteria for patient's selection was the evidence, during follicular monitoring, that one ovary was vaginally unreachable. In some cases, even if the ovaries were seen, they were easier accessible transabdominally. During the execution of the mixed pick up procedure, the same transvaginal probe was used also for transabdominal oocyte retrieval, with the advantages to have a focused framework of observation, high-resolution pictures and to bypass the use limitations of the ecograph instruments exclusively equipped with a vaginal probe.

Among the IVF cycles carried out in the period between January 2012 and December 2017, were identified 21 women who had underwent contemporarily both transvaginal and transabdominal ultrasoundguided follicle aspiration, since oocytes pick up was performed by transvaginal approach in one ovary and via transabdominal aspiration in the contralateral ovary. As shown in Table 1, the total number of oocyte retrieved by transvaginal or transabdominal approach was roughly comparable and no significant differences in the number of mature, degenerated and normally fertilized oocytes were found. 
Table 1: Comparison of in vitro fertilization outcomes between transabdominal and transvaginal follicular aspiration in the same patients

\begin{tabular}{|c|c|c|c|}
\hline Parameters & Transabdominal & Transvaginal & $P$ value \\
\hline Number of oocyte & & & \\
\hline Total & $5 \pm 0.7$ & $8.1 \pm 0.9$ & $\mathrm{~ns}$ \\
\hline Mature & $3 \pm 0.9$ & $6.8 \pm 0.7$ & $\mathrm{~ns}$ \\
\hline Degenerated & $1 \pm 0.06$ & $0.44 \pm 0.19$ & $\mathrm{~ns}$ \\
\hline Normal fertilization & $1.7 \pm 0.5$ & $5.3 \pm 0.6$ & $\mathrm{~ns}$ \\
\hline
\end{tabular}

Note: Value are expressed as mean $\pm \mathrm{sd}$ or percentage

It should be noted that for all transabdominal aspirations, a single ovarian puncture was requested to retrieve all oocytes. This is an important aspect of the present procedure, since in most cases, due to the lesser elasticity of the abdominal wall, the transabdominal follicular aspiration requires multiple ovarian punctures, through different abdominal wall entries, which may cause patient pain and increase the risk of abdominal residual scar or injury to the viscera [13]. Moreover, the oocyte retrieval was completed under the same conscious sedation of patients and no pain killers were usually prescribed to use at home. Furthermore, for all patients and independently by the retrieval method used neither relevant intra-operative complications nor postoperative infections were observed.

In conclusion, transabdominal follicular aspiration can be considered a safe and feasible route for the pick up also in patients undergoing a mixed oocyte retrieval procedure.

\section{References}

1. Feldberg D, Goldman JA, Ashkenazi J, Shelef M, Dicker D, et al. (1988) Transvaginal oocyte retrieval controlled by vaginal probe for in vitro fertilization: a comparative study. J Ultrasound Med 7: 339-343. [Crossref]

2. Feichtinger W, Kameter P (1986) Laparoscopic or ultrasonically guided follicle aspiration for in vitro fertilization. J In Vitro Fert Embryo Transfer 1: 244-249. [Crossref]

3. Hamberger L, Wickland M, Enk L, Nilsson L (1986) Laparoscopy versus ultrasound guided puncture or oocyte retrieval. Acta Eur Fertil 17: 195-198. [Crossref]

4. Wiseman DA, Short WB, Pattinson HA, Taylor PJ, Nicholson SF, et al. (1989) Oocyte retrieval in an in vitro fertilization-embryo transfer program: comparison of four methods. Radiology 173: 99-102.

5. Flood JT, Muasher SJ, Simonetti S, Kreiner D, Acosta AA, et al. (1989) Comparison between laparoscopically and ultrasonographically guided transvaginal follicular aspiration methods in an in vitro fertilization program in the same patients using the same stimulation protocol. J In Vitro Fert Embryo Transf 6: 180-185. [Crossref]
6. Barton SE, Politich JA, Benson CB, Ginsburg ES, Gargiulo AR (2011) Transabdominal follicular aspiration for oocyte retrieval in patients with ovaries inaccessible by transvaginal ultrasound. Fertil Steril 95: 1773-1776. [Crossref]

7. Cohen J, Debacte C, Pez JP, Junca AM, Cohen-Bacrie P (1986) Transvaginal sonographically controlled ovarian puncture for oocyte retrieval for IVF. J In Vitro Fert Embryo Transfer 3: 309-313. [Crossref]

8. Dellenbach P, Nisand I, Moreau L, Feger B, Plumere C, et al. (1985) Transvaginal sonographically controlled follicle puncture for oocyte retrieval. Fertil Steril 44: 656662. [Crossref]

9. Seifer D, Collins R, Paushter D, George C, Quigley M (1988) Follicular aspiration: comparison of an ultrasonic endovaginal transducer with fixed needle guide and other retrieval methods. Fertil Steril 49: 462-467. [Crossref]

10. Roman-Rodriguez CF, Weissbrot E, Hsu CD, Wong A, Siefert C, et al. (2015) Comparing transabdominal and transvaginal ultrasound-guided follicular aspiration: A risk assessment formula. Taiwan J Obstet Gynecol 54: 693-699.

11. O’Shea RT, Forbes KL, Scopacasa L, Jones WR (1988) Comparison of transabdomina and transvaginal pelvic ultrasonography for ovarian follicle assessment in in vitro fertilization. Gynecol Obstet Invest 26: 52-55. [Crossref]

12. Nakagawa K, Ohgi S, Nakashima A, Horikawa T, Saito H, et al. (2009) Laparoscopically-assisted transabdominal oocyte retrieval in an infertility patient with ovarian malposition. Reprod Med Biol 8: 85-87.

13. Edris F, Holiva N, Baghdadi S, Eskandar M, Vilos AG, et al. (2014) Single operator ultrasound guided transabdominal oocyte retrieval in patients with ovaries inaccessible transvaginally: a modified technique. Gynecol Obstet 4: 1000214

14. Damario MA (2002) Transabdominal-transperitoneal ultrasound-guided oocyte retrieval in a patient with Mullerian agenesis. Fertil Steril 78: 189-191. [Crossref]

15. Raziel A, Vaknin Z, Schachter M, Strassburger D, Herman A, et al. (2006) Ultrasonographic-guided percutaneous transabdominal puncture for oocyte retrieval in a rare patient with Rokitansky syndrome in an in vitro fertilization surrogacy program. Fertil Steril 86: 1760-1763. [Crossref]

16. Lavy G, Diamond MP, Nero F, Schark K, DeCherney AH (1987) Transvaginal and transabdominal ultrasound for monitoring of follicular development in an in vitro fertilization and embryo transfer program: patient response. J In Vitro Fert Embryo Transf 4: 293-295. [Crossref]

17. Osemwenkha A, Osaikhuwuomwan J (2016) Transabdominal follicular aspiration in an in vitro fertilization cycle: experiences with an unusual but necessary intervention in a resource-limited setting. Clin Exp Reprod Med 43: 54-57. [Crossref]

18. Deutinger J, Reinthaller A, Csaicsich P, Riss P, Fischl F, et al. (1987) Follicular aspiration for in vitro fertilization: sonographically guided transvaginal versus laparoscopic approach. Eur J Obstet Gynecol Reprod Biol 26:127-133. [Crossref]

19. Sekhon L, Said T, Del Valle A (2014) Percutaneous Transabdominal Oocyte Retrieval Using Vaginal Ultrasound Probe: A Novel, Effective and Safe Method for Oocyte Retrieval in Patients with Vaginally Inaccessible Ovaries. Fertil Steril 101: e26.

Copyright: (C2018 Baldini D. This is an open-access article distributed under the terms of the Creative Commons Attribution License, which permits unrestricted use, distribution, and reproduction in any medium, provided the original author and source are credited. 